\title{
Editorial
}

\section{A New Direction}

\section{ROGER COOTER and VIVIAN NUTTON*}

It is with pleasure and a sigh of relief that we can now report that the Wellcome Trust has expressed its commitment to the continuance of Medical History as an outlet for the best international scholarship in the field. Negotiations are currently in progress as to changes in internal operations that will need to be made in order to secure the health of journal's future. We will report on those negotiations in subsequent issues.

This issue marks the end of one regime and the beginning of another. In September 2010 Caroline Tonson-Rye retired from her post as Assistant Editor. For eighteen years she managed the daily operations of the journal with consummate efficiency, discretion, and grace. The high standards of Medical History could not have been sustained without her. We wish her every happiness in her well-deserved retirement. Michael Laycock now takes over from Caroline to maintain the journal's standards of production and excellence. Among moves to this end, readers will now find that we preface all articles with abstracts and keywords. Other changes to our housestyle can be found on http:// www.ucl.ac.uk/histmed/downloads/Medhist.pdf.

This issue also signals changes in the intellectual aspirations of Medical History. Its articles, addressing the themes of transnational communication, health promotion, forensic medicine, socialism and psychology, and critique of globalisation and visual culture, suggest the wider range of subjects, discourses, and approaches we wish to encourage. While articles across time on the history of disease, institutions, and the professions involved with health and healing are still very much to be welcomed, we particularly invite those that move on historiographically as much as empirically. Indeed, we invite those that through solid historical research contribute to the better understanding of the world in which we now live and of which history writing itself must be regarded as constituent - perhaps rather more self-consciously than in the past. New 'manifestos for history' have been written, and we invite those who focus on health, healing, and the body to engage with them robustly, challenging and extending them. At a moment when the discipline of history and the humanities in general are under threat from forces both economic and intellectual, it is time to up the value of history as a resource in public thinking. In our biologised world, historians of medicine have the privileged expertise to do so. We invite contributors to take full advantage of it.

(C) Roger Cooter and Vivian Nutton, 2011.

* Professor Roger Cooter, The Wellcome Trust Centre for the History of Medicine at UCL, 183 Euston Road, London NW1 2BE, UK.

Email: r.cooter@ucl.ac.uk
Professor Vivian Nutton, The Wellcome Trust Centre for the History of Medicine at UCL, 183 Euston Road, London NW1 2BE, UK. Email: v.nutton@ucl.ac.uk 